\title{
PMU data-Based analysis of power system stability
}

\author{
Li Xiamei, ${ }^{1,}$, Sui Huibin ${ }^{1, b}$ \\ ${ }^{1}$ school of electrical engineering, Shandong university, Shandong 250000, China;

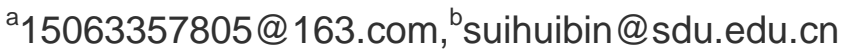

Keywords: Phase angle difference, Generator angle, Stability of the power system.

\begin{abstract}
With the development of PMU(phasor measurement units), further study of the relationship between power system stability and the state variables provides the reference for the monitoring the stability of the system. This paper presents a consistent of phase angle difference change at nodes and the change of generator angle in judging the stability of the system. Phase angle of each node can be measured directly in real-time. Phase angle difference reflects the change of generator status; the phase angle difference changes reflect the network operating status. The change of network state can reflect the stability of power system in real-time. The effectiveness of this method is demonstrated by using the three machines nine nodes system.
\end{abstract}

\section{Introduction}

In recent years, with the gradual increase of electricity demand and the proportion of new energy power generation ${ }^{[1-2]}$, now, the stability of power systems are facing more and more serious problems ${ }^{[3]}$ since 2003. Dozens of large-scale blackout accident ${ }^{[4]}$ has took place across the world, which lead to a serious economic loss and social impact.

Whether the power system suffered from the load fluctuation or network failure is security is important to the security of the whole power system. Transient stability analysis method is mainly based on the numerical simulation ${ }^{[5]}$ and the energy function ${ }^{[6]}$ with the use of direct method about classical model, the method is widely applied ${ }^{[7-9]}$ because it can quickly determine the stability margin of power system. But, this approach relied on the system model of the system largely. The transient potential ${ }^{10]}$ energy distribution is unbounded when the branch included the oscillation center; the kinetic energy cannot be absorbed by the network, which lead to the power system oscillation and instability. If the relative angle difference changes within the bounded range ${ }^{[11]}$, the grid can be thought of transient stability; otherwise, transient instability, this is a sign of power grid failure. Literature [12] use the method of angle - speed trajectory, which reflects a general steady state of the whole power grid, but it contains a lot of useless information.

In this paper, by analyzing the simple "source - net - load" system after disturbance, whether the tendency of the change of the node phase angle difference to a constant is used to evaluate the stability of power system after a disturbance. Also, node angle difference change is consistent with the tendency of generator rotor angle. Monitoring the change of the phase angle difference provides the reference for the monitoring the stability of the system .PMU can directly measure the node phase angle.

\section{2 the illustration of the "source - net - load" system}

Figure 1 shows the simple "source-network-load" model. It discusses the process of the transmission line from failure to the removal of the fault, which includes dynamic characteristics of generator, motor and power system.

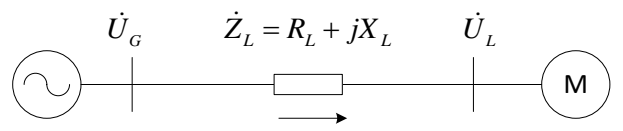

Fig 1 the simple "source-network-load" model

Figure 2 shows the simple "source - net - load" mathematical model. Generator has potential 
$\dot{E}$ 、 internal impedance $\dot{Z}_{G}$, voltage $\dot{U}_{G}$ and the voltage at the end of the line $\dot{U}_{L}$.

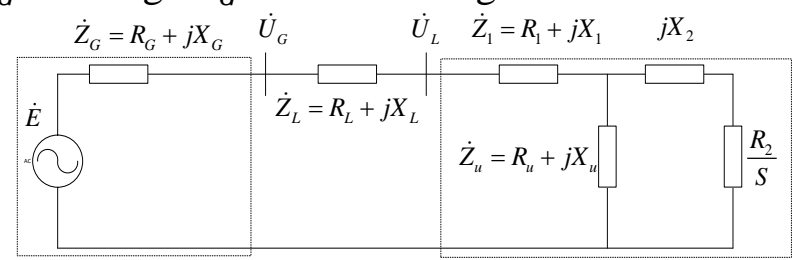

Fig 2 the simple "source-network-load" system model

Generator model as shown in formula (1):

$$
\left\{\begin{array}{l}
\frac{d \delta}{d t}=(w-1) w_{0} \\
\frac{d w}{d t}=\frac{P_{m}-P_{e}}{T_{j G}}
\end{array} .\right.
$$

Angle $\delta$, angular velocity for generator is $\omega$, the mechanical power is $P_{m}$, electromagnetic power is $P_{e}$, the inertia constant is $T_{j G} . \dot{E}(1)=1.1, P_{m}=0.3608$, the model of the motor is shown below:

$$
\left\{\begin{array}{l}
T_{j M} \frac{d S}{d t}=T_{m M}-T_{e M} \\
T_{e M}=\frac{2 T_{e M \max }}{S / S_{c r}+S_{c r} / S}\left(\frac{\dot{U}_{L}}{\dot{U}_{L N}}\right)^{2} \\
T_{m M}=k\left[\alpha+(1-\alpha)(1-S)^{k m}\right] \\
\dot{Z}_{D}=R_{1}+j X_{1}+\frac{\left(R_{u}+j X_{u}\right)\left(R_{2} / S+j X_{2}\right)}{R_{u}+j X_{u}+R_{2} / S+j X_{2}} \\
S=\left(w_{s}-w\right) / w_{s}
\end{array} .\right.
$$

Mechanical torque is $T_{m M}$; electromagnetic torque is $T_{e M}$; the maximum electromagnetic torque is $T_{e \text { Mmax }}$; the rated voltage is ; critical slip is $S_{c r}$; the rating voltage is $U_{L N}$; the actual voltage is $U_{L}$; static torque is $\alpha$; mechanical properties load index is $\mathrm{km}$; the load rate of the motor is $k$; Each particular value of the parameters as shown in formula (3).

$$
\left\{\begin{array}{l}
R_{1}+j X_{1}=0.0465+j 0.295 \\
R_{2} / S+j X_{2}=0.02 / S+j 0.12 \\
R_{u}+j X_{u}=0.35+j 3.5 \\
S_{0}=0.014 \\
S_{c r}=0.0625 \\
T_{e M \max }=1.282 \\
k=0.56 \\
\alpha=0.15 \\
k m=2
\end{array} .\right.
$$

\subsection{The rule of the node angle difference change}

The angle between the potential of the generator and the generator terminal voltage as the rotor angle. Figure 3 and figure 4 shows the different disturbance $\mathrm{N}=4$ and the angle change curve when $\mathrm{N}=16$, figure 3 shows when $\mathrm{N}=4$ generator failure occurs in 20 seconds.increases, in 100 seconds to remove failure, Angle to reduce and gradually become stable. Figure 4 Angle curve shows that with the increase of impedance is after 100 seconds to remove the fault Angle has been reduced, instability of a pendulum. 


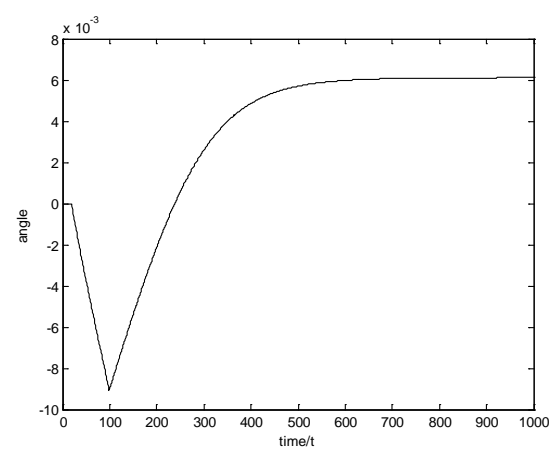

Fig $3 \quad \mathrm{~N}=4$ generator rotor angle curve

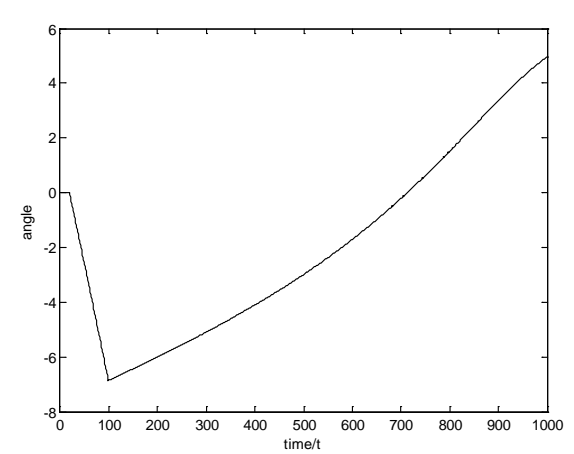

Fig $4 \quad \mathrm{~N}=16$ generator rotor angle curve

\subsection{The rule of the node angle difference change}

The bus voltages and angles can be measured by the PMU, but the angle of the generator and the angular acceleration need some indirect way of measuring or some electromagnetic sensors.

$$
k=\frac{\theta_{G}-\theta_{L}}{\Delta t} .
$$

The phase angle for $U_{G}$ is $\theta_{G}$; the phase angle for $U_{L}$ is $\theta_{L} ; \theta_{G}$ minus $\theta_{L}$ is node angle difference; $\Delta t$ is sampling step length. Figure 5 shows phase angle difference curve, which rises at 20 seconds and reduces at 100 seconds with the increase of impedance, $\mathrm{k}$ tends to zero, the system is stable. Figure 6 shows the phase angle difference curve oscillation, and finally, k can't tend to zero, instability occurs.

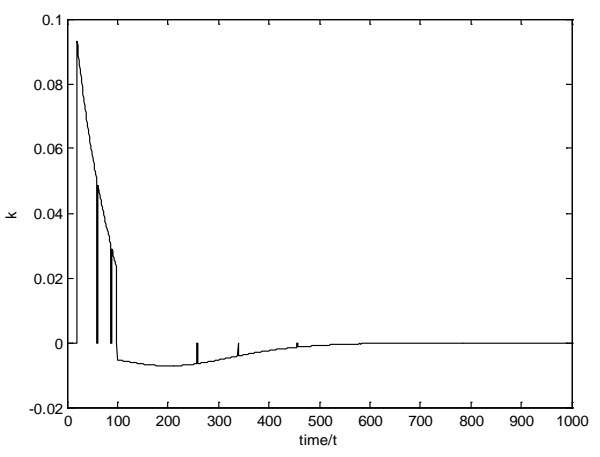

Fig $5 \mathrm{~N}=4$ node angle difference change

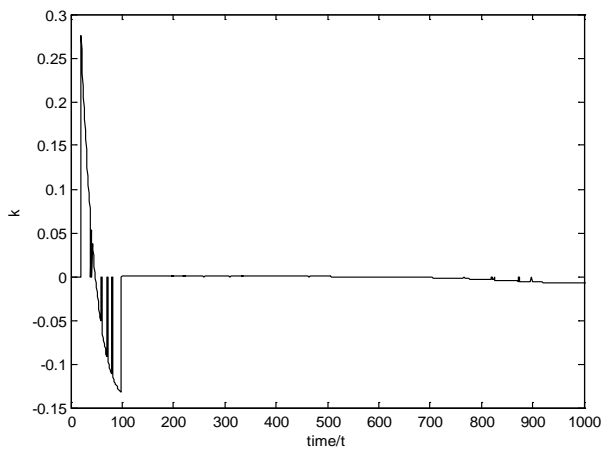

Fig $6 \mathrm{~N}=16$ node angle difference change

The system move to balance point, eventually, to a new stable equilibrium when transient stability; the curves of bus voltage phase angle difference eventually converges to zero. Otherwise, the curve will persists oscillate when the power system is transient instability.

\subsection{Angle difference and $k$ is consistent on stability judgement}

$$
\begin{aligned}
& P(\delta)=\frac{U_{i} U_{j}}{X_{i j}} \sin \delta_{i j} . \\
& P(\delta)=\frac{E_{i} U_{j}}{X_{i j}} \sin \delta_{i j} .
\end{aligned}
$$

$\delta_{i j}$ is node phase angle difference in formula (5) and $\delta_{i j}$ is generator rotor angle in formula (6). Angle difference reflects the change of generator rotor status, the phase angle difference changes reflect the network operating status.

\section{Case Studies}

\subsection{3-machine 9-bus system}

All generators are used in classical model, excitation system is considered, the over-speed protection system of the unit itself is ignored. The stability and instability of different scenarios are used to validate the effectiveness and adaptability of this method by PSAT simulation. The sampling 
step length is 0.01 seconds. The simulation results as shown in the figure below:

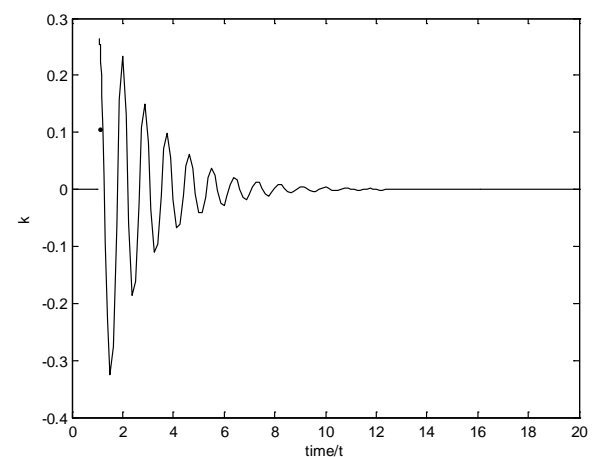

Fig 7 Bus 5 virus bus 2 change of $\mathrm{k}$

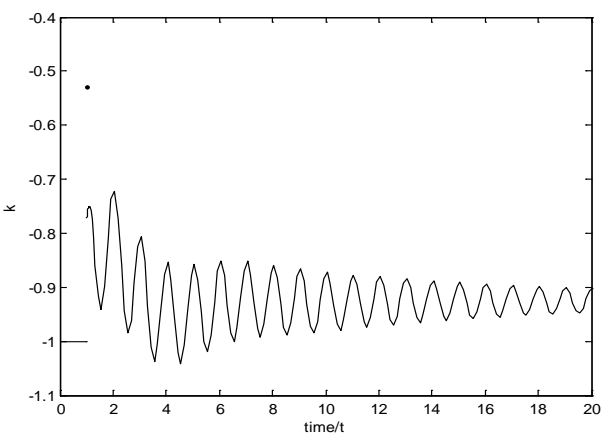

Fig 9 Bus 5 virus bus 2 change of $k$

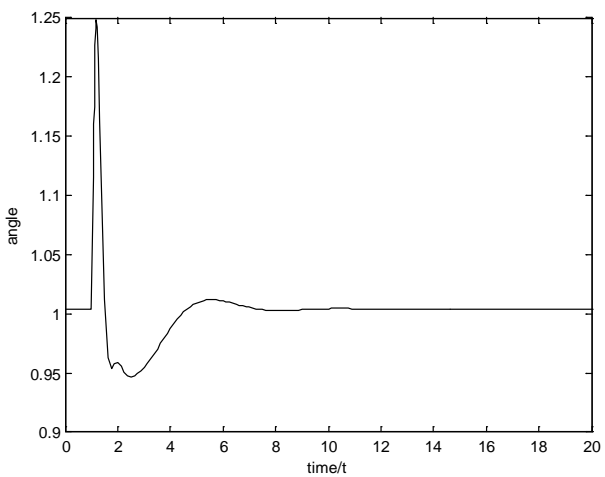

Fig 11 Generator rotor angle under stability

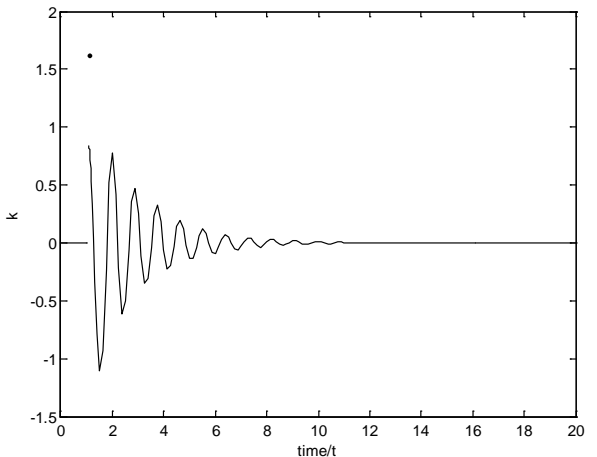

Fig 8 Bus 4 virus bus 2 change of $\mathrm{k}$

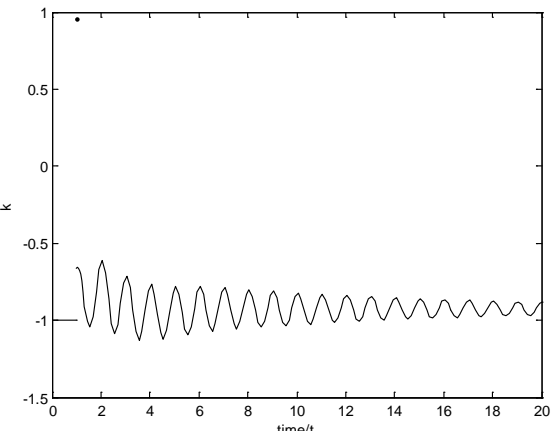

Fig 10 Bus 7 virus bus 1 change of $\mathrm{k}$

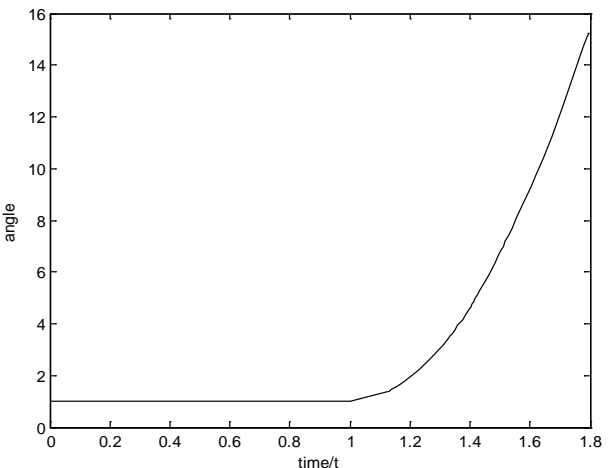

Fig 12 Generator rotor angle under instability

Figure 7 and figure 8 show k has the largest fluctuations when a failure occurs in 1 seconds, the system persists oscillation to zero in 1.2 seconds around the origin after resection; in the end, the system is in steady state. Figure 9 and figure 10 show that k fluctuation is serious and finally can't tend to a constant; the system is in unstable state with the increase of fault clearance time under the same fault. Figure 11 shows generator rotor angle tends to a constant under stability; Figure 12 shows generator rotor angle tends to infinity under instability. Figure 7 and figure 8 correspond to Figure 11; Figure 9 and figure 10 correspond to Figure 12.

\section{Conclusion}

Angle difference reflects the change of generator status; the change of the phase angle difference reflects the network operating status. Phase angle difference information can directly reflect the transient stability of the system. PMU can get the whole voltage phase angle difference curve in real time. From above, the phase angle difference and angle change trend after disturbance is consistent with power system stability. 


\section{References}

[1] SUN Hongbin, GUO Qinglai, PAN Zhaoguang. Energy internet: concept, architecture and frontier outlook [J].Automation of Electric Power Systems, 2015, 39(19):1-8.

[2] LIU Ji Zhen. Basic issues of the utilization of large-scale renewable power with high security and efficiency [J].Proceedings of the CSEE, 2013, 33(16):1-8.

[3] BAO Yanhong, FENG Chang you, XU Taishan. Online Security and stability comprehensive auxiliary decision-making of Power system [J]. Automation of electric power systems, 2015, 39 (1):104-110.

[4] FANG Yong jie. Application of emergency control to reduce risk of system collapse triggered by power transmission interface tripping: thinking on the India power blackouts [J]. Automation of Electric Power Systems, 2013, 37(4):1-6.

[5] YU Yixin, Chen Liyi, The security and stability of power system [M] Science press, 1998, Beijing.

[6] Fouad A, Vital V Power system transient stability analysis using the transient energy function method [M] Prentice Hall, 1992.

[7] WANG Chengshan, YU Xuyang. Transient stability probability analysis method based on fitting curve [J].Automation of Electric Power Systems, 2003, 27(6):5-9.

[8] SONG H, KEZUNOVIC M. Stability control using PEBS method and analytical sensitivity of the transient energy margin[C] //IEEE PES Power Systems Conference.

[9] LU Jinling, JI Qunxing, ZHU Yongli. Power grid vulnerability assessment based on energy function [J].Power System Technology, 2008, 32(7):30-33.

[10]Meng Xiangxia, Liu Mingguang. The distribution mechanism analysis of potential energy based on network structure [J] Automation of Electric Power Systems, 2016.

[11] Gu Zhuoyuan, Tangyong, The emergency control scheme of transient stability based on relative kinetic energy [J] Automation of Electric Power Systems, 2014.

[12]GU Zhuoyuan, Tangyong, Sun Huadong, The transient Angle stability estimation method based on the trend of rotation difference virus Angle difference.[J] Proceedings of the CSEE, 2013, 33(31): $65-72$ 